\title{
The improvement of a piezoelectric thin film measuring instrument
}

\author{
Da-Long Cheng ${ }^{1}$ 、Yu-Che Wang ${ }^{1}$ Kuo-Sheng Kao ${ }^{1, *}$ 、Chia-Lin Chen ${ }^{2}$, Wei-Tsai Chang ${ }^{2}$ \\ ${ }^{1}$ Department of Computer and Communication, Shu-Te University, Kaohsiung city, 82445 Taiwan \\ ${ }^{2}$ Metal Industries Research and Development Center, Kaohsiung city, 81160 Taiwan \\ *Corresponding author. +886-7-6158000 ext. 4818, E-mail address: kks@stu.edu.tw
}

\begin{abstract}
The evolution of a piezoelectrical films using charge direct coupling is developed in this study. The servo motor has initial taken for generating a fixed force, however, the bearing condition of the motor is sometimes out of condition. As a result, the servo motor has been supplanted by stepper motor because of its stability and precision. Extensive memory costs and clock cycles for a microcontroller are prevented by the circuit's function simplification. The noise is suppressed and cleared by specific filter adopted. The various conditions of piezoelectrical thin film samples have been tested and analyzed. Furthermore, several times of testing has executed for inspecting and verifying the system stability.
\end{abstract}

Key words: noise suppression 、 Arduino 、 amplifier

\section{Introduction}

There are many unwanted noises appeared when measuring an extreme weak signal of any electronic circuits. These noises may cause from signal transmission process, the capacitive or inductive effect of the circuit itself, the mutual oscillation of the components and the direct coupling from the power source. The noise can cause very serious interference under the case of extreme weak signals measurement. This work is tried to observe a pulse signal of peak value of less than $20 \mathrm{mV}$ just as like piezoelectrical effect of a thin film. The pulse signal will be detected and then process with microcontroller for analyzing. The limitations of extreme weak signal amplification and noise suppression schemes will be carried out.

\section{Experiment and set-up}

Figure 1 shows a schematic of Arduino nano adopted in this study. Since the resolution of Arduino microcontroller like the ATmega 328 is 210 , the deviation value of $4.9 \mathrm{mV}$ is calculated with the measurement range from 0 to $5 \mathrm{~V}$. The sampling rate of the general ATmega analog to digital converter (ADC) function is default setting the prescaler value of 128. It takes 13 working cycles for running an $\mathrm{ADC}$ function. As the fundamental oscillation frequency of the ATmega is $16 \mathrm{MHz}$, the sampling rate of the ADC function is calculated to be 9600 $\mathrm{Hz}$.

A pulse signal of peak value of $20 \mathrm{mV}$ and duration of $1 \mathrm{~ms}$ has been taken as source signal. The amplifier and peak detector circuit are designed and manufactured for measuring the source signal. The source signal is amplified by the amplifier circuit to the detectable range of the ATmega. The peak detecting circuit is then response for measuring and registering the peak value. Furthermore, the amplifier is a non-ideal circuit; a bias eliminating circuit must be additionally designed at the output stage. Also, the buffer stage located within the sampling and readout circuit can avoid interference between the stages. The flow chart of the system operation is shown in Fig. 2.
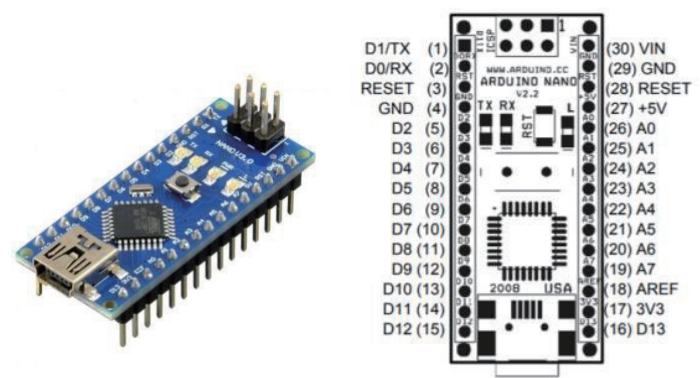

Figure 1 Arduino nano schematic

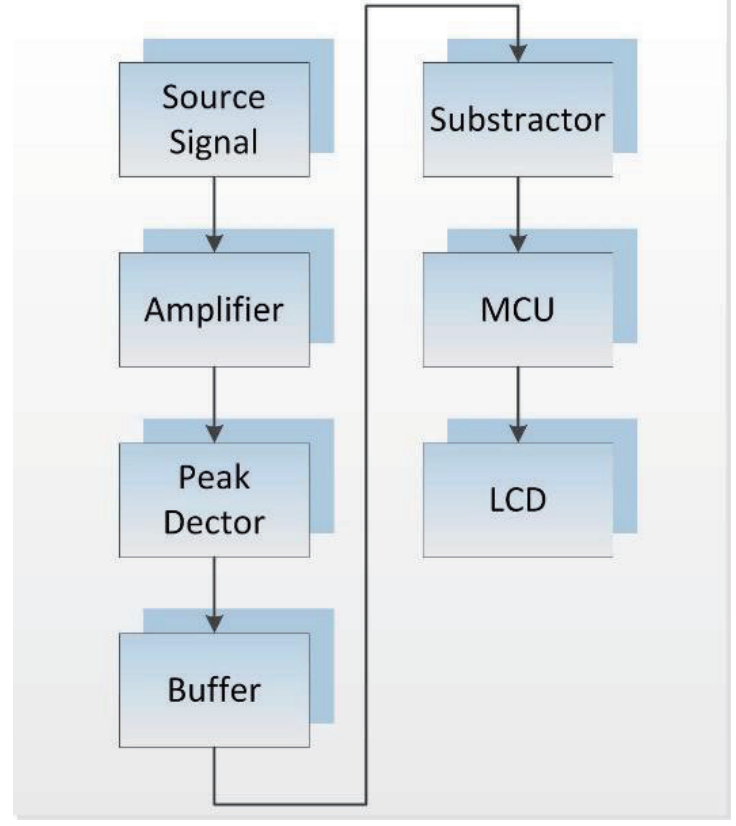

Figure 2 - Flow chart of operation 
According to the configuration of the resistances, the amplifier is setting as a non-inverting with the magnification of 150. The amplifier and lump elements arrangements are shown in Fig. 3.

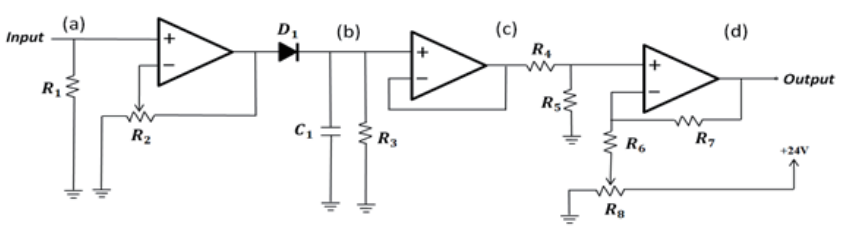

Figure 3 - technical drawing of system circuit

The extreme weak signal is therefore can be magnified to cross over the potential voltage of the diode. A peak value detector is working under the diode be forward-biased. The capacitor here has been charged when magnified signal storage. The source and the amplified signal have shown in Fig. 4. It's clear that the extreme weak signal has been transferred as a stable DC voltage.

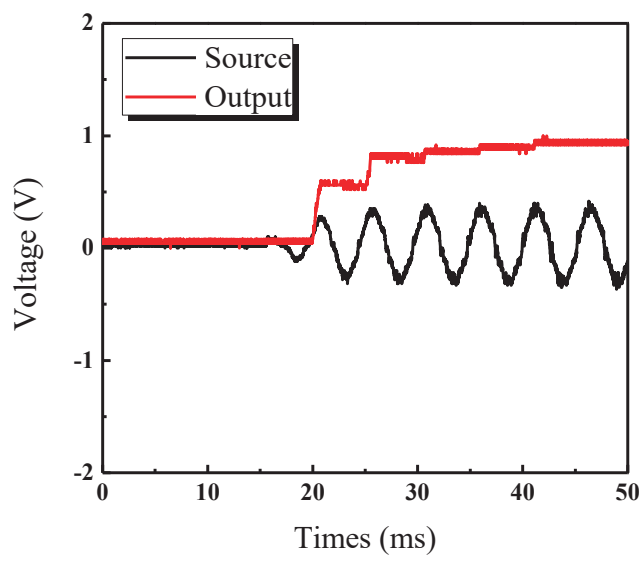

Figure 4 The input and output signal of peak value detector

However, an extra DC voltage will be superimposed with the output signal under the condition of the magnification over 100 . Therefore, a subtractor is adopted for reducing the unwanted DC voltage. Each step of signal processed with peak value detector, buffer and subtractor is shown as Fig. 5 8.

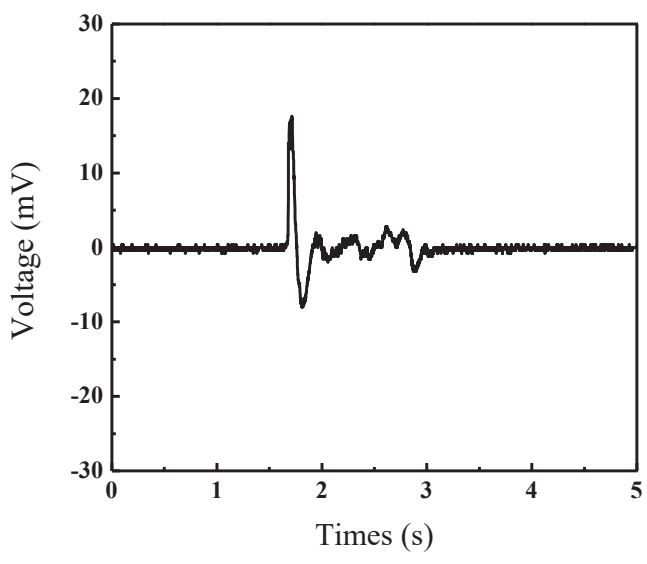

Figure 5 v source signal

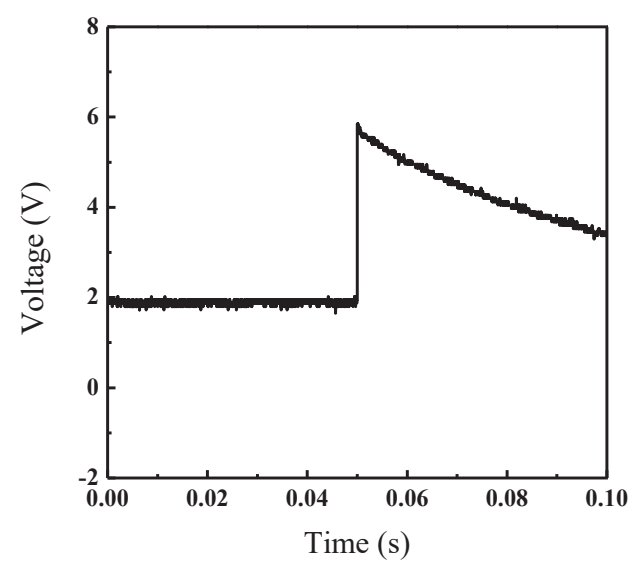

Figure 6 - signal processed with peak value detector

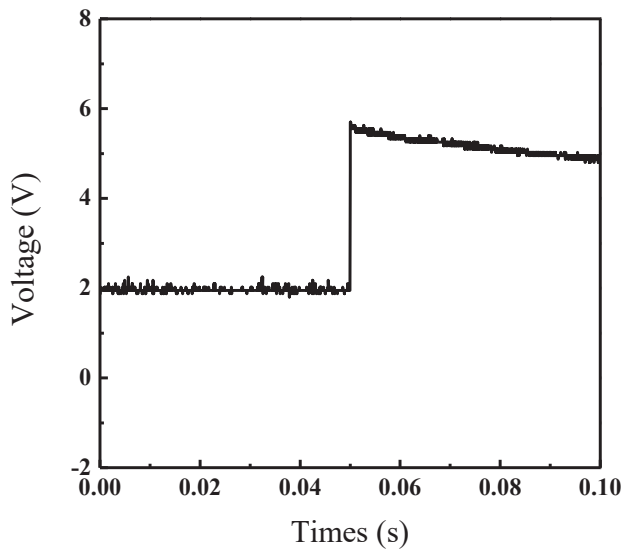

Figure 7 、 signal processed with buffer 


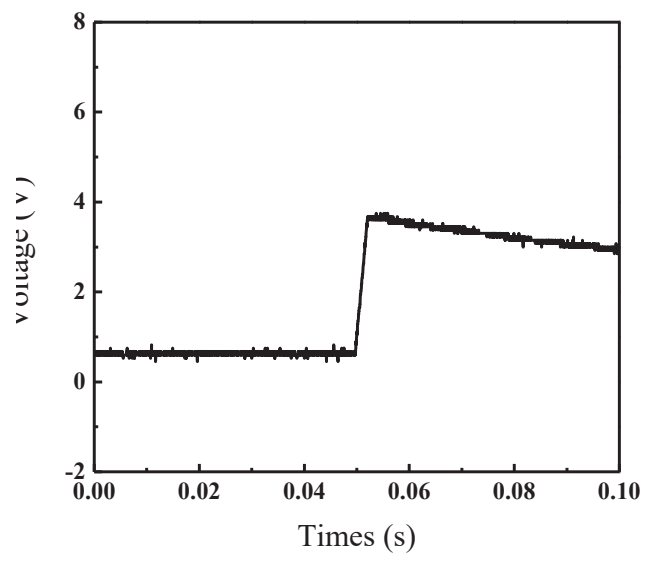

Figure 8 、 signal processed with subtractor

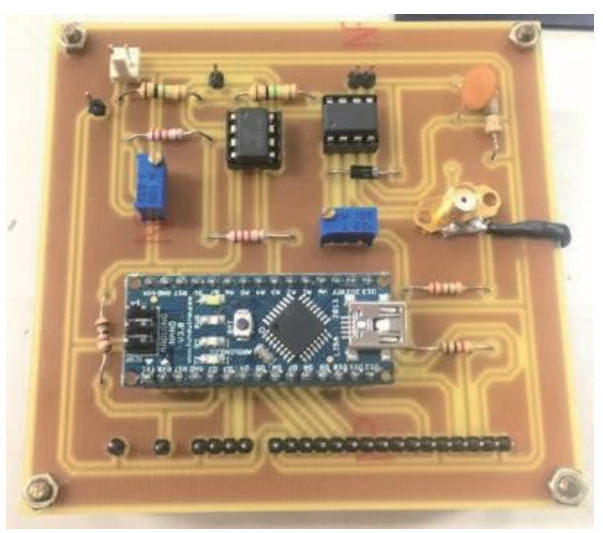

Figure 9、 PCB Layout

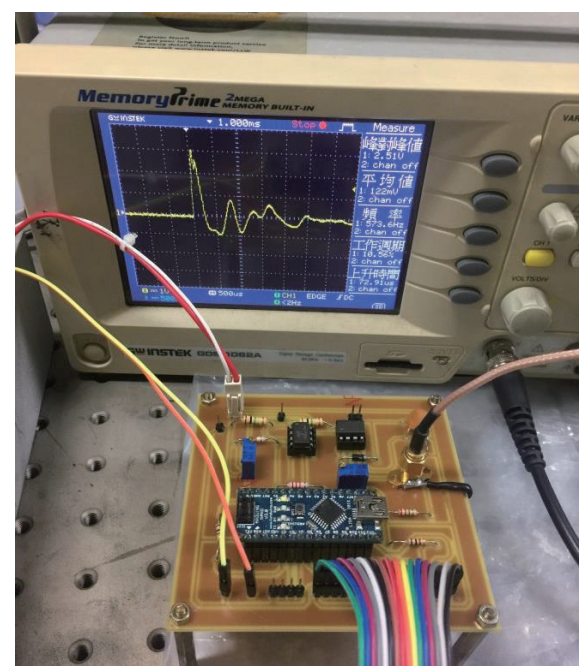

Figure 10、actual system product

\section{Conclusion}

It can be observed from Fig. 5 to Fig. 8 that the origin source signal with peak value of $20 \mathrm{mV}$ and duration of $0.5 \mathrm{~ms}$ has been amplified and detected. The signal reveals a longer duration since it has been amplified. The buffer and subtractor process the signal in quick succession. Finally, a faultless output signal is generated for converting by MCU.

It is worth to mention that some unknown signals will be introduced or mutual coupled for the case of using solderless breadboard. The situation comes better when perf board is adopted for connected, however, the origin extreme weak signal still be laid over unavoidable.

Finally, the printed circuit board (PCB) layout is realized to solve the noises. Although a few space noises are still mutual coupled, the observation and amplification of the origin extreme weak signal through the system is success.

\section{Acknowledgements}

The authors acknowledge financial supports of , Industrial Development Bureau, Ministry of Economic Affairs and Medical Devices and Metal Industries Research and Development Center.

\section{References}

[1]. J. F. Shepard, P. J. Moses, S. Trolier-McKinstry, "A technique for the measurement of $\mathrm{d} 31$ coefficient of piezoelectric thin films", Mat. Res. Soc. Symp., Boston, USA (1996) pp. 225-230.

[2]. M. A. Dubois and P. Muralt, "Measurement of the effective transverse piezoelectric coefficient $\mathrm{e}_{31, \mathrm{f}}$ of $\mathrm{AlN}$ and $\mathrm{Pb}\left(\mathrm{Zr}_{\mathrm{x}}, \mathrm{Ti}_{1-}\right.$ х) $\mathrm{O}_{3}$ thin films", Sensors and Actuators A: Physical, 77 (1999), pp. 106-112.

[3]. Q. Guo, G. Z. Cao, and I. Y. Shen, "Measurements of piezoelectric coefficient $\mathrm{d}_{33}$ of lead zirconate titanate thin films using a mini force hammer", Journal of Vibration and Acoustics, 135 (2013) 011003.

[4]. K. Rittenmyer and P. Dubbelday, "Direct Measurement of the Temperature-Dependent Piezoelectric Coefficients of Composite Materials by Laser Doppler Vibrometry," J. Acoust. Soc. Am., 91 (1992), pp.2254-2260.

[5]. C. Chao, Z. Wang, and W. Zhu, "Measurement of Longitudinal Piezoelectric Coefficient of Lead Zirconate Titanate Thin/Thick Films Using a Novel Scanning Mach-Zehnder Interferometer," Thin Solid Films, 493 (2005), pp.313-318. 
Recent Developments on Information and

Communication Technology (ICT) Engineering- Meen, Yang \& Zhao ISBN: 978-981-14-2136-5 